\title{
Effect of Tris(1,3-Dichloroisopropyl) Phosphate as Flame Retardant on Mechanical Properties and Fire Resistivity of PU Composites
}

\author{
Amamer Musbah Redwan ${ }^{1,2} *$, Khairiah Haji Badri ${ }^{2}$ and Azizah Baharum ${ }^{2}$ \\ ${ }^{1}$ Department of Chemistry, Faculty of Science, Bani Waleed University, PO Box 5338 Tripoli, Libya. \\ ${ }^{2}$ School of Chemical Sciences and Food Technology, Faculty of Science and Technology \\ University Kebangsaan Malaysia, 43600 UKM Bangi, Selangor-Malaysia. \\ * Corresponding author: amamer1982@yahoo.com
}

\begin{abstract}
Palm oil based polyurethane composites that are reinforced with Tris(1,3- dichloroisopropyl) phosphate TDCPP which function as flame retardants (FR) at different proportions of 3,6 and 9\% were synthesized. The polyurethane composite's influence on the mechanical (tensile strength, impact and flexural limits and modulus) and thermal characteristics (fire test) of the polyurethane composites were examined. Increased loading of TCPP resulted in increased hardness (up to 39\%) which translates to a marginal increase in Shore D hardness as the TDCPP content increases. With higher TDCPP loading, the impact and flexural capabilities decreased because of the fragile interfacial bonding between the TDCPP and PU matrix. Loading of 3\%, 6\% and 9\% of TDCPP resulted in the decrease of impact strength by $25 \%, 24 \%$ and $23 \%$ respectively. Lowest flexural ability (at a reduction of 21\%) was exhibited with the highest amount of TDCPP loading (9\%) and the modulus had decreased by $23 \%$. When the percentage of TDCPP loading increased, this resulted in a lower heat of combustion of the composites which manifested in the form of lower burning rate from $5.4 \mathrm{~mm} / \mathrm{s}$ to $2.7 \mathrm{~mm} / \mathrm{s}$ in the fire test operated.
\end{abstract}

[DOI: 10.22401/ANJS.22.1.05]

Keywords: Fire test, TDCPP, Flame retardant, Mechanical analysis, Polyurethane composite.

\section{Introduction}

Polyurethane is a high molecular weight polymer based on the polyaddition of polyfunctional hydroxyl group and isocyanate. There are two types of polyhydroxyl compounds commonly used particularly polyester and polyether polyols $[1,2]$. Polyester (PE) is a high molecular weight substance which contain ester group as a repeating unit in the chain. The mechanical characteristics and morphological structure of polyurethane depend mainly on the polyol structure, molar mass and its functionality and to a lesser extent, on the nature of the polyisocyanate [2]. The most important commercial PU products are foams that are generally branched as either rigid or flexible depending on their mechanical properties and cross-link densities [3]. Rigid polyurethane foams are widely used in building insulation and domestic appliances, due to their superior mechanical characteristics and low density. Polyurethane (PU) can be produced by using diverse techniques for example, prepolymerization, single-step polymerization and quasi-prepolymerization [4]. Single-step polymerization occurs when diisocyanate, polyol and catalyst are combined and the chain augmentation happens in one stage. The heat released makes this system most appropriate to make thin items. Quasi-prepolymerizatio technique uses the reaction between polyol and excess diisocyanate. Thus, the urethane prepolymer consists of higher free isocyanate of $15 \%$ to $30 \%$ and is known as isocyanate quasiprepolymers or semiprepolymer since the diisocyanate particles have partially reacted with the polyol [5]. Addition of blowing agents and other additives like flame retardants are also important. Polyurethane composites are used for \{several\} years in many applications like craft, house and marine structures, mass transport systems, automotive industries, construction and building. The expertise gained altogether these applications demonstrated that the in-service behavior of a compound composite extremely depends on the performance of the reinforcing particlematrix or endless fiber-matrix interface that contributes to fret transfer.

Flame retardant is a type of materials that are blended into plastics to have certain defined reactions all along combustion. Those reactions cause the initially flammable 
substances to ignite with more difficulty and will constrain the propagation compared to the original substrate under laboratory test conditions [6]. There are two major classes of flameproof chemicals; additive flame retardation and reactive flame retardation Additive flame-retardants are the nice majority of the flame retardation. They embrace halogenated flame retardants. They include halogenated flame retardants [7], organophosphorus and nitrogen-based organic flame retardants Phosphites are the type of decomposed hydroperoxide secondary antioxidants which work s flame retardant. Organic phosphite is used widely as affirmer to any polymer type and to keep its physical polymer nature such as its color and molecular weight during processesing. Activity such as hydroperoxide decomposition decreased due to its increasing ability in receiving electron and its group size attach to phosphate and blocked aryl phosphite [8]. The focus of the present study is to enhance the mechanical properties and importantly the fire resistivity of PU composites by using TDCPP as an additive.

\section{Materials and Method}

The palm oil-based ester as the raw starting material was prepared and characterized as reported by Badri et al. [9] and was mixed with catalyst (tetramethylhexanediamine, TMDHA), surfactant Niax L5404 and 3,5-dirtbutylphenyl phosphite as flame retarding agent. 2,4-diphenylmethane diisocyanate (MDI) was then reacted with the PKO-based ester resin with a mixing ratio of $1: 1$. The mixture is agitated vigorously using a standard propeller with a speed of $2000 \mathrm{rpm}$ for 10 seconds at room temperature, placed in a waxed cavity plate and passed at ambient temperature for $20 \mathrm{~min}$ with load pressure of $760 \mathrm{MPa}$. Finally the polyurethane (PU) sample was conditioned for 16 hours at $23 \pm 2^{\circ} \mathrm{C}$ before further characterization.

\subsection{Experimental Procedure MechanicalCharacterizatios}

In order to determine the hardness of a sample, a Portable shore D hardness indentor (Durometer Affri system Series 3300 MRS, Affri Cee-Versea, Italy) was utilized based on measurement according to the ASTM D2240 standards. The boards produced were made into samples with dimensions of $130 \mathrm{~mm}$ length $\mathrm{x} 130 \mathrm{~mm}$ width $\mathrm{x} 3 \mathrm{~mm}$ thickness. The mean obtained from the measurements of five specimens was used as the value of the shore D hardness. ASTM D256-88 standard was also used to carry out impact testing. Izod technique was carried out on un-notched samples with dimensions $63 \mathrm{~mm}$ length $\mathrm{x} 13$ $\mathrm{mm}$ width $\mathrm{x} 3 \mathrm{~mm}$ thickness on a Zwick impact tester (Model 5101, Zwick Roell Group, Atlanta, Georgia, United State of America ) with a 2 Joule (J) pendulum energy. The strength of impact on the tester by crosssectional areas $\left(\mathrm{mm}^{2}\right)$ of the specimens was calculated by dividing the energies (Joule). The values of the obtained impact strengths signified the mean measurements of five specimens. Flexural strength was tested by using three-point bending test which was carried out according to the ASTM D790-86 standard. The samples obtained were cut to test samples with dimensions of $130 \mathrm{~mm}$ length x $13 \mathrm{~mm}$ width $\mathrm{x} 3 \mathrm{~mm}$ thickness. Next, a flexural test was done by utilising a Universal Test Machine (Model5525, Instron Corporation, Norwood, Massachusetts, United State of America) at a cross-head speed of 3.1 $\mathrm{mm} / \mathrm{min}$. The values obtained signified the mean measurements of five specimens.

\subsection{Fire test}

Fire test was done to conclude the relative burning qualities and flame resistance. It explains and measures the reaction of products, assemblies or materials reactions to flame and heat under controlled laboratory environment. The test results show the glowing and flaming time in seconds for all the materials that were tested. The ASTM D 5048-90, Procedure B which was plaque specimens test was carried out.

\section{Result and discussion}

\subsection{Mechanical properties}

The results achieved from the mechanical tests are presented in Table (1). Fig.(1, 2, 3 and 4). The results indicated that, for TDCPPfilled PU composites, the higher the TDCPP content, the lower the flexural and impact strength were. This could be due to the fact 
that the presence of TDCPP leads to an increase in polymer brittleness, as observed in work carried out by Dvir et al. 2003 [10] mentioned that the effect of FR on polypropylene composites lead to decrease in the impact and flexural strength and modulus of polymer.

Table (1)

The mechanical properties of the control PU and TDCPP-filled PU composites.

\begin{tabular}{|c||c||c||c|c|}
\hline $\begin{array}{c}\text { TDCPP } \\
\text { content, } \\
(\mathbf{W t} \text {. \%) }\end{array}$ & $\begin{array}{c}\text { Hardness } \\
\text { Index Shore } \\
\mathbf{D}\end{array}$ & $\begin{array}{c}\text { Impact } \\
\text { Strength } \\
\left(\mathbf{K J} / \mathbf{m}^{\mathbf{2}}\right)\end{array}$ & $\begin{array}{c}\text { Flexural } \\
\text { Strength } \\
(\mathbf{M P a})\end{array}$ & $\begin{array}{c}\text { Flexural } \\
\text { modulus } \\
(\mathbf{M P a})\end{array}$ \\
\hline \hline $\mathbf{0}$ & 38 & 7.0 & 33.8 & 1029 \\
\hline \hline $\mathbf{3}$ & 42 & 6.3 & 31.1 & 969 \\
\hline $\mathbf{6}$ & 48 & 6.0 & 23.8 & 857 \\
\hline $\mathbf{9}$ & 56 & 5.7 & $21 / 7$ & 825 \\
\hline
\end{tabular}

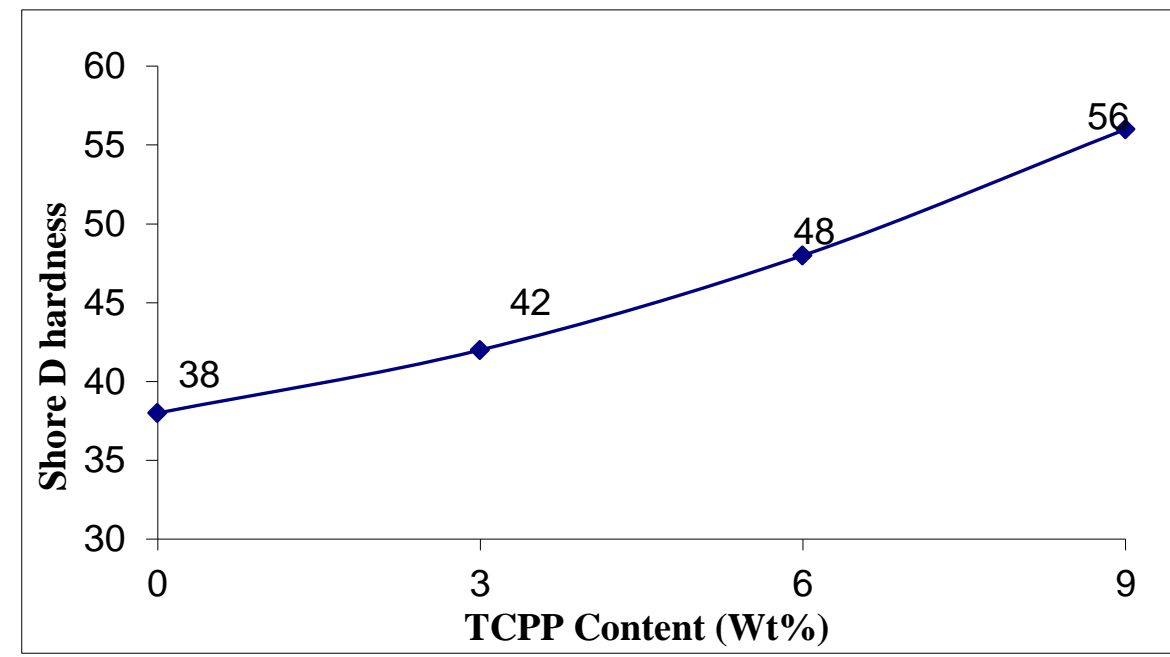

Fig.(1): The Shore D hardness index of the control PU and TDCPP-filled PU composites.

The plot of Shore D hardness index as shown in Fig.(1). Indicated the effect of varying the amount of TDCPP in the PU on the shore D hardness index. The hardness is most commonly defined as the resistance of polymer material to indentation. Indentation is the pressing motion on a point at the polymer sample with known force [11].

The hardness index provides an early indicator to physical strength of the PU composites. The hardness of the index will increase with the increase in the quantity of filler. The finding of this study is similar to the findings obtained by Rusu et al. (2001) [13] and Badri et al. (2006) [11] whereby the composites with added filler produced greater hardness compared to those without filler. This study showed that the hardness index increased by $8 \%$ with 3 wt $\%, 27 \%$ with 6 wt $\%$ and $38 \%$ with $9 \mathrm{wt} \%$ of TDCPP loading.

Fig.(2) and Table (1) display the impact strength of TDCPP-filled PU composites and the controlled PU. The impact strength of materials revealed their ability to resist fracture. As the TDCPP content increased, the impact strength of the TDCPP-filled PU composites were reduced. This happened due to the weakening interfacial bonding between the PU matrix and TDCPP when larger quantity of TDCPP were utilised. The highest impact strength obtained for the controlled PU was $6.9 \mathrm{k} / \mathrm{m}^{2}$. The PU composite with $6 \%$ TDCPP has the lowest impact strength when compared with others. The impact strength decreased by $9 \%$ with $3 \%, 13 \%$ with $6 \%$ and $17 \%$ with $9 \%$ TDCPP loading. Optimum 
loading was noticed at 3\% TDCPP. The presence of higher loading of TDCPP in the polymer matrix caused decreasing ability to absorb impact energy. The TDCPP caused matrix discontinuity and each particle was a site of stress concentration that led to microcrack [9]. The findings showed that PU composites had a higher impact strength than TDCPP.

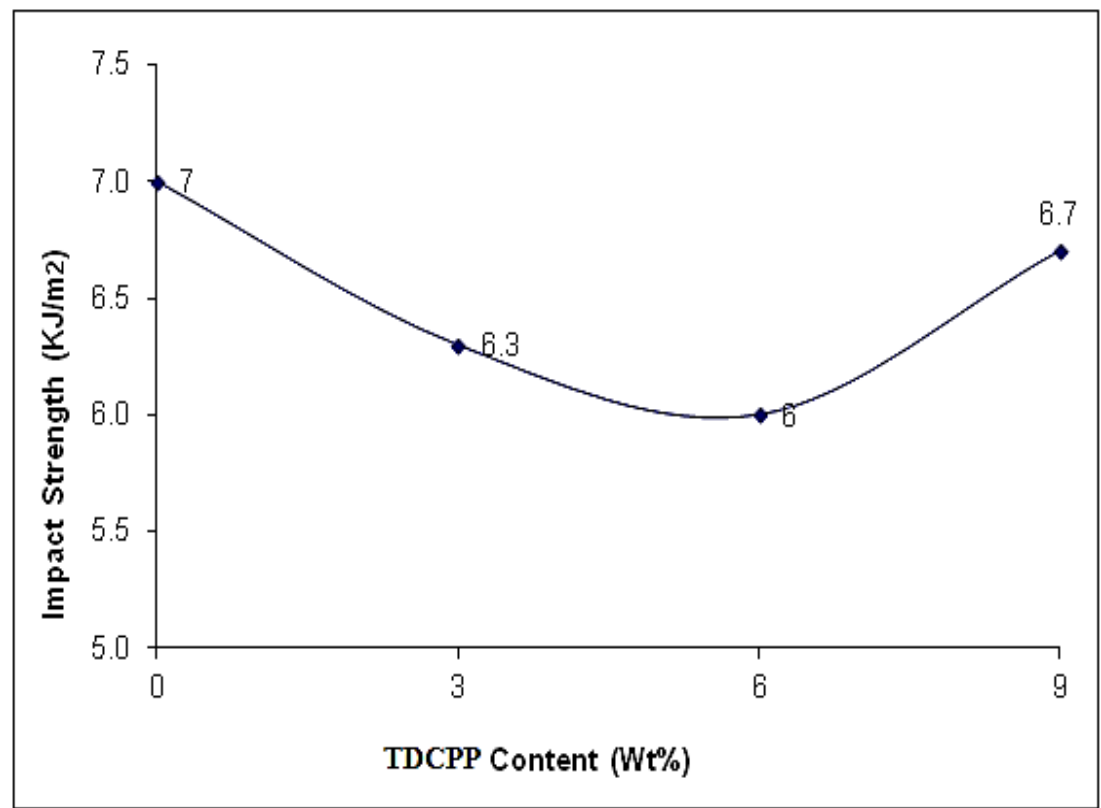

Fig.(2): The impact strength of the control PU and TDCPP-filled PU composites.

Fig.(3) and Fig.(4), show the flexural strength and modulus of the control PU and the TDCPP-filled PU composites respectively. As the content of TDCPP increased, the flexural strength decreased. The reduction in strength was ascribed to the poor adhesion between TDCPP and PU matrix due to agglomeration of TDCPP particles. The agglomeration become more dominant at higher content of TDCPP and the flexural strength reduced due to uneven dispersion of TDCPP throughout the PU matrix. This result is in agreement with the study reported by Jang et al. (1998) [14] where when phosphate TDCPP increased, the flexural strength of polymer composite decreased. The maximum flexural strength of polymer composite was observed on control PU as summarized in Table (1) and reduced by $6 \%$ with addition of $2 \%$ TDCPP. Further reduction was observed with higher amount of FR (4\% gave a reduction of $25 \%$ from the control PU). The highest amount of TDCPP gave the lowest flexural strength as shown in Fig.(3). 


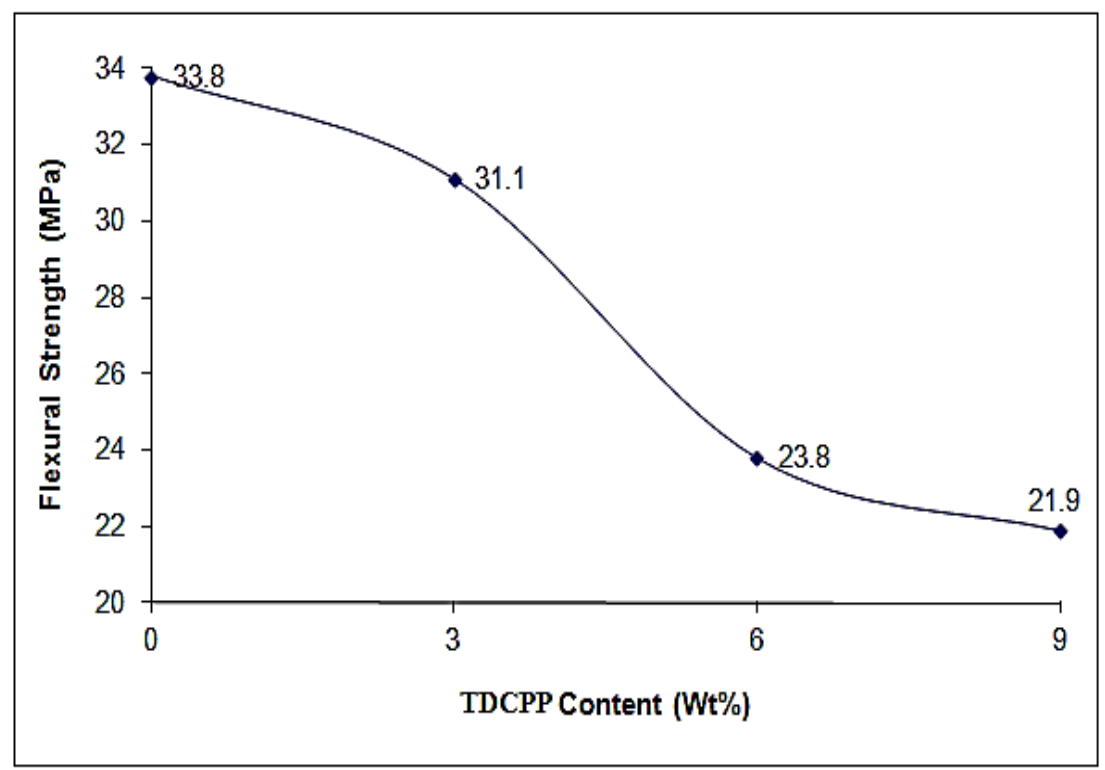

Fig.(3): The flexural strength of the control PU and FR-filled PU composites.

Fig.(4), described the flexural modulus of the control PU and the PU composites. The flexural modulus reflects the rigidity of the material. The modulus decreased by $5 \%, 16 \%$ and $19 \%$ respectively when $2 \%, 4 \%$ and $6 \%$ of TDCPP were added to the PU matrix. The lowest modulus was observed at $6 \%$ TDCPP with modulus of $827 \mathrm{MPa}$. Higher loading of
TDCPP decreased the wetting properties of the matrix and reduced the degree of encapsulation of matrix around the flame retardant. As such, the TDCPP was exposed to direct stress and low stress transfer was experienced.

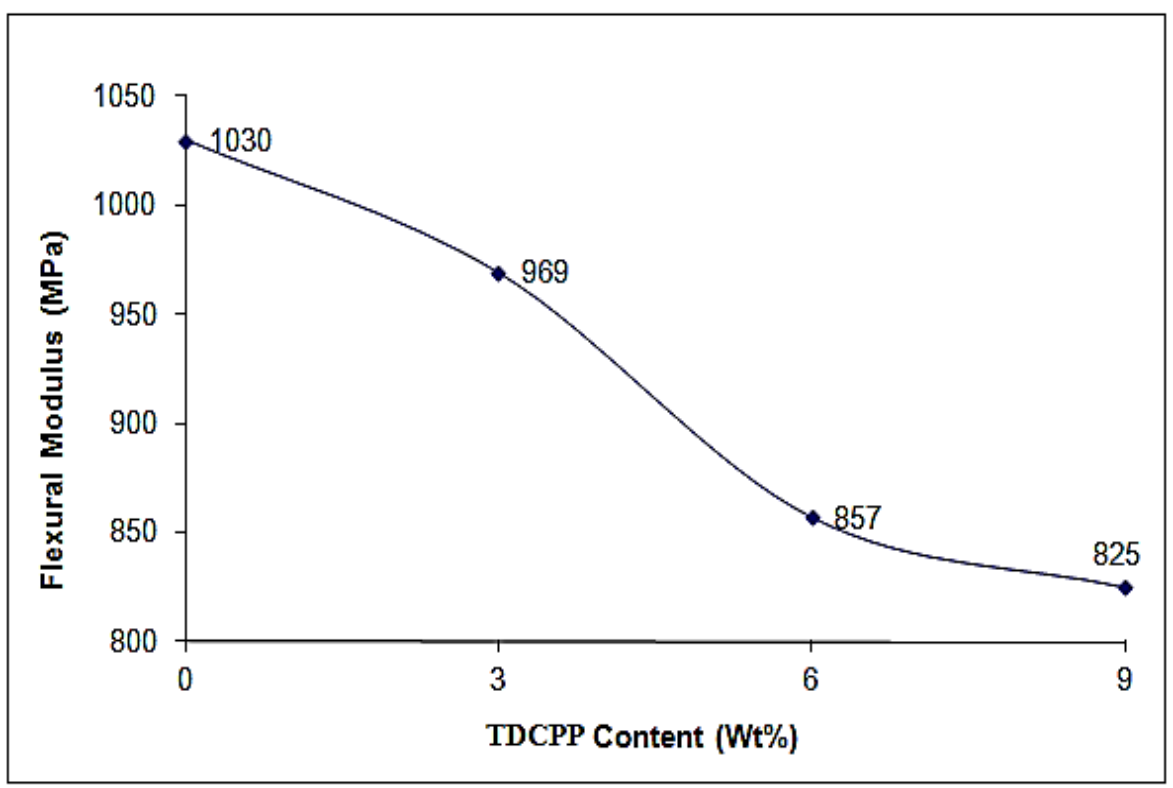

Fig.(4): The flexural modulus of the control PU and TDCPP-filled PU composites.

\subsection{Fire resistivity test}

The PU burnt fast and was totally razed after being exposure to the flame. The rate of burning of the TDCPP-filled PU composite was lower than the control PU. This is caused by the high flammability of PU that is highly combustible without the presence of flame retardant [15] as displayed by Fig.(4). The rate of burning of the PU composites reduced significantly by $26.6 \%$ when 2 wt $\%$ of TDCPP was added. A similar trend in the reduction of burning rate was detected for PU composite with a $4 \mathrm{wt} \%$ of TDCPP content. 
It was decreased by $21.5 \%$ the PU composite with $6 \mathrm{wt} \%$ TDCPP has the lowest burning rate it was decreased by $17.7 \%$. Similar to previous studies, the TDCPP has a good flame retardant effect on the PU composites [16]. The flame retardant dissociate into radical element which compete with chain propagating and branching steps during the combustion process. One of the most damaging element in the oxidation process is the hydroperoxide, $\mathrm{ROOH}$. Under rising temperature hydroperoxides decomposed through hemolytic cleavage to produce two free radicals.

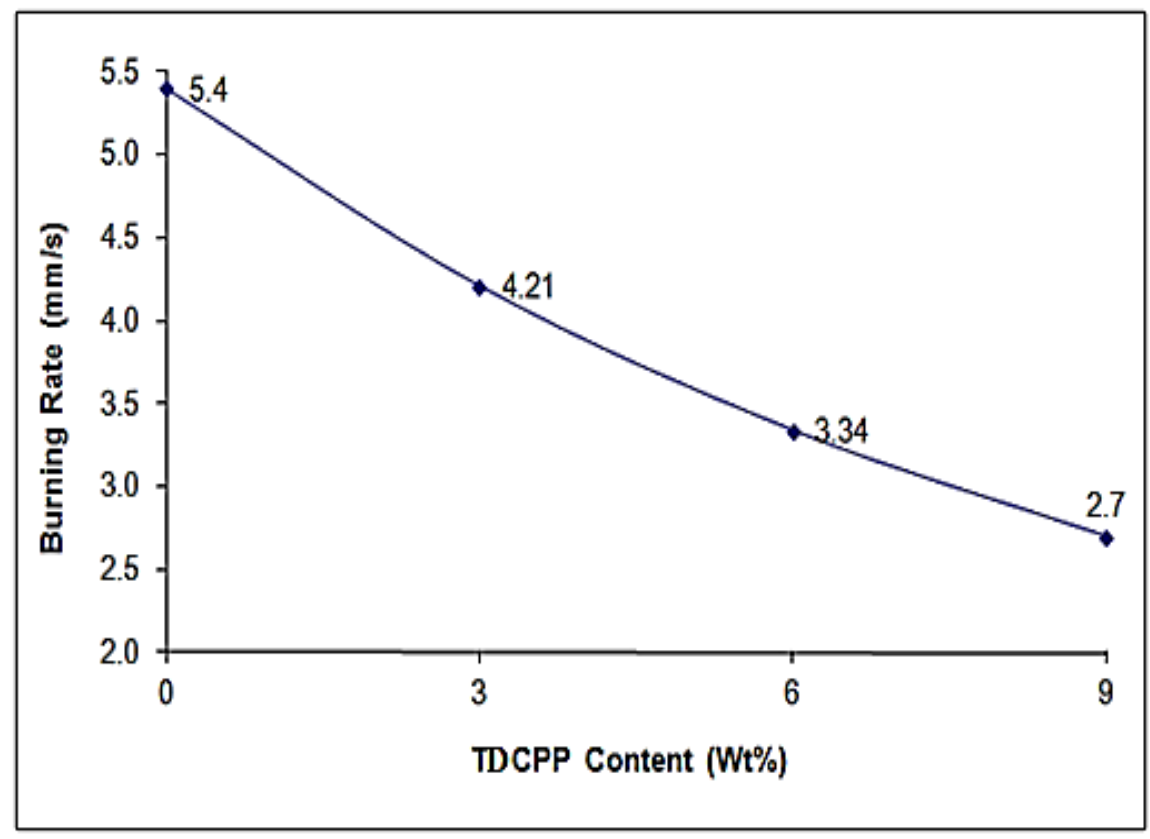

Fig.(5): Burning rate of control PU and FR-filled PU Composites.

This step demonstrates the catalytic nature of autoxidation. The destruction of hydroperoxides, which continually build up in the polymer, is essential in protecting the polymer Phosphites prevent further formation of free radicals by decomposing unstable hydroperoxides prior to their homolytic cleavage. Instead, the unstable hydroperoxide forms a stable product [17].

\section{Conclusion}

Tris (1,3-dichloroisopropyl)phosphate TDCPP which act as flame retardant (FR) were examined and its influenced on the mechanical (tensile strength, impact and flexural limits and modulus) and thermal characteristics (fire test) were deliberated. 3\%, $6 \%$ and $9 \%$ (by weight) of TDCPP content were used in the study. When the amount of filler increased, the results of study on the mechanical properties exhibited an obvious increase in the hardness index. An increase of $9 \%, 28 \%$ and $39 \%$ in the hardness index was noted with a loading of TDCPP (weight) at $3 \%, 6 \%$ and $9 \%$ respectively. With the increased levels of TDCPP content, the impact strength of the TDCPP-filled PU composites dropped. The reason being the weakening of the interfacial bonding between the filler and the PU matrix as the content of TDCPP increased. The control PU $\left(7 \mathrm{~kJ} / \mathrm{m}^{2}\right)$ had exhibited the highest impact strength. At a $9 \%$ TDCPP content the PU composite was showing lowest impact strength as compared to the other test subjects. The highest amount of TDCPP gave the lowest flexural strength (reduction of $33 \%$ for $9 \%$ TDCPP). The flexural modulus reflects the rigidity of the material. The modulus decreased by $5 \%, 16 \%$ and $19 \%$ when $3 \%, 6 \%$ and $9 \%$ of TDCPP were added to the PU matrix respectively. The lowest modulus was observed at 6\% TDCPP. Higher loading of TDCPP decreased the wetting properties of the matrix and reduced the degree of encapsulation of matrix around the flame retardant. The fire test indicated lower burning rate (from $5.30 \mathrm{~mm} / \mathrm{s}$ to 2.80 $\mathrm{mm} / \mathrm{s}$ ) as the percentage loading of TDCPP increased. 


\section{Acknowledgment}

The principal researcher would like to thank the Department of Chemistry and Faculty of Sciences, Bani Waleed University for facilities and services given. The authors wish to thank Polymer Research Centre, Universiti Kebangsaan Malaysia for all facilities offered and the Malaysia Ministry of Science, Technology and Innovation for finance and facilities given.

\section{References}

[1] Khairiah Haji B., Amamer M R., Effect of phosphite loading on the mechanical and fire properties of palm-based polyurethane, Sains Malaysiana, 39.5, 769-774, 2010.

[2] Clemitson, I. R. Castable Polyurethane Elastomers. Taylor and Francis. (New York): CRC Press, 2015.

[3] Redwan A., Haji Badri K., Tarawneh M., The Effect of Aluminium Hydroxide (ATH) on the Mechanical Properties and Fire Resistivity of Palm-Based Fibreboard Prepared by Pre-Polymerization Method, Advance Material Research,_1087, 287-292, 2015.

[4] Wong, C. S. \& Badri, K. H. Chemical Analyses of Palm Kernel Oil-Based Polyurethane Prepolymer. Scientific Research Publishing, (3), 78 - 86, 2012.

[5] Guilleminot J., Kondo D., Binetruy C., Panier, L. Hariri., Krawczaka, P, A micromechanical analysis of a local failure criterion for particle-reinforced composites, Composite Science \& Technology, 67, 2384-2389, 2007.

[6] Dedecker, Kristof, Luca Binaghi, and Mauro Corio., Process for Making Rigid Polyurethane or Urethane-Modified Polyisocyanurate Foams. U.S. Patent Application 15/112, 742. 2015.

[7] Frank W., Polymer additive analysis by pyrolysis-gas chromatography II. Flame retardant, Journal Chromatography, 8, 225235, 2000.

[8] Lyons J., The chemistry \& uses of fire retardants, New York: Wiley-Interscience, 1970.

[9] Redwan A., Khairiah Haji B., Azizah B., A urethane block copolymer as binder for fireresist palm-based fibreboard, Polymers \& Polymer Composites, 24, 9: 681-687, 2016.
[10] Dvir H., Gottlieb M., Daren S., Tartakovsky S. Optimization of a flameretarded polypropylene composite, Composite Science \& Technology, 63, 1865-1875, 2003.

[11] Badri K.., Mat Amin Z., Othman H., Abdul Manaf K., Khalid K., Effect of fillerto-matrix blending ratio on the mechanical strength of palm-based biocomposite boards, Polymer International, 55,2, 190195, 2006.

[12] Dowling N E., Mechanical behavior of materials, 2nd ed. Upper Saddle River, NJ: Prentice Hall, 1999.

[13] Rusu R., Sofian N., Rusu D., Mechanical and thermal properties of zincpowder filled high density polyethylene composites, Polymer Test, 20, 409-417, 2001.

[14] Jang J., Hyuksung C., Myonghwan K., Hyunje S., The effect offlame retardant on the flammability and mechanical properties of paper-sludge/phenolic composite, Polymer Test, 19, 1, 269-279, 1998.

[15] Reed S., Jonathan P., Kiven T., Polyurethane/poly [bis (carboxilatophenoxy phosphazene blends and their potential as flame retardant materials, Polymer Engineering Science, 40, 2, 350355, 2000.

[16] Salamon J C., Polymeric material encyclopedia, flame retardants (overview)," Boca Raton, London: CRC Press 1996.

[17] Cheremisinoff N. P., Handbook of engineering polymeric materials, $2^{\text {nd }}$ edition, USA:CRC Press/Lewis publishers, 105, 1997. 